$\underline{\text { Preprint typeset in JHEP style - HYPER VERSION }}$

IFUP-TH/2007-13

UT-Komaba/07-10

YITP-07-34

\title{
QCD String as Vortex String in Seiberg-Dual Theory
}

\author{
Minoru Eto ${ }^{*, \S, a}$, Koji Hashimoto ${ }^{\dagger, b}$ and Seiji Terashima ${ }^{\ddagger}, c$ \\ * INFN, Sezione di Pisa, Largo Pontecorvo, 3, Ed. C, 56127 Pisa, Italy \\ $\S$ Department of Physics, \\ University of Pisa, Largo Pontecorvo, 3, Ed. C, 56127 Pisa, Italy \\ $\dagger$ Institute of Physics, the University of Tokyo, Komaba, Tokyo 153-8902, Japan \\ $\ddagger$ Yukawa Institute for Theoretical Physics, \\ Kyoto University, Kyoto 606-8502, Japan \\ ${ }^{a}$ E-mail: minoru@df.unipi.it \\ ${ }^{b}$ E-mail: koji@hep1.c.u-tokyo.ac.jp \\ c E-mail: terasima@yukawa.kyoto-u.ac.jp
}

\begin{abstract}
We construct a classical vortex string solution in a Seiberg-dual theory of $\mathcal{N}=1$ supersymmetric $S O\left(N_{c}\right)$ QCD which flows to a confining phase. We claim that this vortex string is a QCD string, as previouly argued by M. Strassler. In $S O\left(N_{c}\right)$ QCD, it is known that stable QCD strings exist even in the presence of dynamical quarks. We show that our vortex strings are stable in the Seiberg-dual theory.
\end{abstract}




\section{Contents}

1. Introduction and summary 1

2. $S O\left(N_{c}\right)$ theory and its supersymmetric vacua 3

3. Vortex string solution

4. Stability of the vortex string solution 9

\section{Introduction and summary}

Understanding of confinement in QCD is one of the long-standing problems in particle physics. Quarks are confined, and force between them develops a linear potential described by a confining non-Abelian flux tube, which is called QCD string. This flux tube is expected to be described by dual Meissner effect. In the Meissner effect, magnetic flux is confined due to condensation of electrically charged fields (Higgs fields), which is well described by Abelian-Higgs model. The magnetic vortex string solution in that model is called ANO(Abrikosov-Nielsen-Olesen) vortex [1].

The duality used in this argument, which mimics the electric-magnetic duality in Maxwell theory, should be generalized to a non-Abelian duality. The renowned Seiberg's duality [2, 3] provides a proper basis for addressing this problem, in $\mathcal{N}=1$ supersymmetric non-Abelian gauge theories. In some situations of the non-Abelian Seiberg duality, the dual "magnetic" theory is weakly coupled at low energy while the original "electric" theory is strongly coupled, thus classical ANO-like strings may be constructed in the magnetic theory, as a concrete realization of the QCD string. In this paper, we construct a classical non-BPS vortex string solution in a Seibergdual of $\mathcal{N}=1$ supersymmetric $Q C D$ with non-Abelian gauge groups which flows to a supersymmetric confining phase at low energy. As for construction of classical non-Abelian vortex strings in the spirit of studying the QCD strings, see [4, 5, 6, (7).

There is an interesting coincidence. This Seiberg's dual theory which is IR free was used recently [8] to reveal that in fact in $\mathcal{N}=1$ supersymmetric QCD there is a meta-stable supersymmetry-breaking vacua, in addition to the supersymmetrypreserving vacua. Here "QCD" means non-Abelian gauge theories with "quark" matter fields in the vector (fundamental) representation of the gauge group. Our previous paper [9] studied solitons in these meta-stable vacua. In particular, we have 
shown there that for the dual of the $S U\left(N_{c}\right)$ QCD there is no vortex string, while for the dual of the $S O\left(N_{c}\right)$ gauge theories with $N_{f}$ flavors (the dual gauge group is $S O(N)$ with $\left.N=N_{f}-N_{c}+4\right)$, there are vortex strings. (For $U\left(N_{c}\right)$ gauge theories, there are vortex strings associated with the $U(1)$ subgroup.) In view of the problem of the QCD strings, is the presence of these vortex strings in the meta-stable vacua just a coincidence?

In fact, these vortex strings in the meta-stable vacua are actually directly related to strings in supersymmetric vacua, in the following way. The meta-stable vacua in [8] are obtained when all the quarks obtain masses. If we tune the masses for the $N_{f}$ quarks in the electric theory as $m=\left(\mu^{2}, \cdots, \mu^{2}, 0, \cdots, 0\right)$ where the first $N$ entries are nonzero, the meta-stable vacua restore supersymmetries, as described in [9]. The non-BPS vortex solutions with these tuned mass parameters in the supersymmetric vacua have the same form as those found in [9]. So, vortex strings found in [9] are expected to be dual of the QCD strings.

In order to identify our classical vortex strings with the QCD strings, there are two issues. One is the stability, and the other is the phase. The following are resolution of these issues. The first issue is that in the real QCD the QCD strings are unstable. Long strings can break via a pair creation of a quark and an antiquark, so any infinitely long string cannot be stable, in the presence of dynamical quarks. To evade this difficulty, in this paper we consider non-Abelian gauge group $S O\left(N_{c}\right)$ instead of usual $S U(3)$ QCD. In $S O\left(N_{c}\right)$ gauge theories, Wilson loops in the spinor representation can be defined, and associated QCD strings are stable because they cannot be broken by quarks lying in the vector representation. This is consistent with our findings in [9]; only for $S O\left(N_{c}\right)$ gauge groups, we found nontrivial topological charges for the vortex strings. $\left(U\left(N_{c}\right)\right.$ gauge theories can accommodate strings, but they are asymptotically non-free because of the crucial $U(1)$ factor necessary for the vortices to live*.) Furthermore, as is well-known, Seiberg's duality for $S O(2)$ gauge theory with no flavor, $N_{f}=0$, reduces to the electric-magnetic duality in the Maxwell theory (the dual group is $S O(2) \sim U(1)$ ), thus the $S O(N)$ series of the duality is not special but naturally shows up.

The other issue is the phase. We have to make sure that the electric (original) theory is in the confining phase at low energy, so that the theory actually has the confining QCD strings. The recipe for this has been studied by M. Strassler [10] (see also [11, 12]) who first developed the idea of this identification of the QCD strings with the vortex strings in Seiberg-dual of $S O\left(N_{c}\right)$ QCD. Our procedures for a confining phase is as follows. First we explicitly construct a classical vortex solution in the dual $S O(N)$ theory at low energy, with the quark masses (in the electric theory) arranged as above. The theory is in confining phase at low energy due to

*This $U(1)$ is obtained by gauging the $U(1)_{\text {Baryon }}$ global symmetry which is common for electric and magnetic theories. Consequently, the electric theory has the same $U(1)$ gauge symmetry and is asymptotically non-free. 
the monopole condensation [3]. Thus our classical vortex string can be naturally identified as a QCD string, because our solutions are string-like objects which carry magnetic flux in the theory Seiberg-dual to the confining gauge theory. The vortex string solution has the tension of the scale $\mu^{2}$, and is reliable for $\mu^{2}<\Lambda^{2}$ where $\Lambda$ is the scale at which the magnetic $S O(N)$ theory is strongly-coupled. The usual QCD string should have the scale of $\Lambda$, thus accordingly we bring $\mu$ to be large and closer to the scale $\Lambda$. For large $\mu, N$ quarks are massive and decoupled, resulting in the electric theory with $N_{f}-N=N_{c}-4$ flavors whose supersymmetric vacuum is in a confining phase at energy lower than $\Lambda$ [3]. This final procedure, at the same time, brings the tension of our vortex string to roughly equal to that of the QCD string. Note that bringing $\mu$ to the large value requires a large gauge coupling constant of that energy scale, via renormalization group. There our tree-level analysis of the vortex soliton solutions is not valid, but they are topologically protected and are expected to remain for large $\mu$. Our procedure relates the classical vortex strings in the magnetic theory (which sat at free magnetic phase at $\mu=0$ ) with the confining phase (large $\mu$ ).

In the following, we will present a classical vortex string solution in the Seibergdual of the $\mathcal{N}=1$ supersymmetric $S O\left(N_{c}\right)$ QCD with $N_{f}$ quarks in the vector representation. When $N_{f}=N_{c}-2$, the dual (magnetic) theory has the gauge group $S O\left(N_{f}-N_{c}+4\right)=S O(2) \sim U(1)$, and we will find a vortex string solution as a direct analogue of the well-known ANO string solution in the Abelian-Higgs model. This is consistent with the the topological argument of [9] that the vortex strings have a $Z$ charge. Our fluctuation analysis will show that our vortex string is stable classically. For generic $N \geq 3$, the topological charge is $Z_{2}$ as shown in [9]. There we will show that a special embedding of the ANO string solution exists.

\section{2. $S O\left(N_{c}\right)$ theory and its supersymmetric vacua}

We consider a Seiberg-dual of the $S O\left(N_{c}\right) \mathcal{N}=1$ supersymmetric QCD with $N_{f}$ quarks in the vector representation of $S O\left(N_{c}\right)$. The matter content of the dual magnetic theory for generic dual gauge group $S O(N)$ with $N=N_{f}-N_{c}+4$ is [2]

\begin{tabular}{c|cccc} 
& $S O(N)$ & $S U\left(N_{f}\right)$ & $U(1)^{\prime}$ & $U(1)_{R}$ \\
\hline \hline$\Phi_{\left[N_{f} \times N_{f}\right]}$ & 1 & $\square$ & -2 & 2 \\
$\varphi_{\left[N \times N_{f}\right]}$ & $\square$ & $\square$ & 1 & 0
\end{tabular}

For $\frac{3}{2}\left(N_{c}-2\right) \geq N_{f} \geq N_{c}-2$, the magnetic theory is IR free and in the so-called free magnetic phase, which we shall make use of. (For $N_{f}=N_{c}-3$ or $N_{c}-4$, the theory is confining, and so later we shall introduce quark mass terms to move from the free magnetic phase the confining phase.) The Kähler potential, the superpotential and 
the D-term potential are

$$
K=\operatorname{Tr}\left[\varphi^{\dagger} \varphi\right]+\operatorname{Tr}\left[\Phi^{\dagger} \Phi\right], \quad W=h \operatorname{Tr}\left[\varphi^{T} \Phi \varphi-M_{q} \Phi\right], \quad V_{D}=\frac{g^{2}}{2} \sum_{A}\left|\varphi_{i}^{\dagger} T_{A} \varphi_{i}\right|^{2}
$$

The symmetric $N_{f} \times N_{f}$ matrix $M_{q}$ is the quark mass matrix in the electric theory. The theory resembles O'Raifeartaigh model, and, in fact, when all the quarks in the electric theory have the same non-zero masses,

$$
M_{q}=\operatorname{diag}\left(\mu^{2}, \cdots, \mu^{2}\right)
$$

there is a meta-stable vacuum in the magnetic theory. The flavor symmetry $S U\left(N_{f}\right) \times$ $U(1)^{\prime}$ is broken down to $O\left(N_{f}\right)$ because of the quark mass term. The vacuum of this theory, meta-stable supersymmetry-breaking one and the one with supersymmetries dynamically restored, were studied in detail in [8]. The meta-stable supersymmetrybreaking vacuum is given by

$$
\Phi=0, \quad \varphi=\left(\begin{array}{c}
\varphi_{0} \\
0
\end{array}\right), \quad \text { with } \varphi_{0}=\mu 1_{[N \times N]}
$$

The vacuum expectation value $\varphi_{0}$ gives color-flavor locking. The vacuum has a cosmological constant, $V_{\min }=\left(N_{f}-N\right)\left|h^{2} \mu^{4}\right|$.

We are interested in supersymmetric vacua which are directly accessible from this meta-stable vacuum, to relate our vortex solutions obtained in [9] with objects in supersymmetric vacua. As described in the introduction, and as already studied in our previous paper [9], if we align the quark masses in the electric theory as

$$
M_{q}=\operatorname{diag}\left(\mu^{2}, \cdots, \mu^{2}, 0,0,0, \cdots, 0\right)
$$

in which only the first $N_{0}$ entries are non-zero with $N_{0} \leq N$, then the meta-stable vacuum restores supersymmetries perturbatively, and is identified with the supersymmetric vacuum of the theory. (If $N_{0}>N$, perturbative vacua in which our vortices live are the supersymmetry-breaking meta-stable vacua which are not of our interest in this paper.) With this choice of the quark masses, the "rank condition" in [8] is satisfied, thus the cosmological constant of course vanishes. The supersymmetric vacuum is

$$
\Phi=\left(\begin{array}{cc}
0 & 0 \\
0 & \Phi_{0}
\end{array}\right), \quad \varphi=\left(\begin{array}{c}
\varphi_{0} \\
0
\end{array}\right)
$$

where $\Phi_{0}$ is arbitrary constant symmetric matrix with the size $\left(N_{f}-N_{0}\right) \times\left(N_{f}-N_{0}\right)$, and the diagonal $N \times N$ matrix $\varphi_{0}$ is

$$
\varphi_{0}=\operatorname{diag}\left(\mu^{2}, \cdots, \mu^{2}, 0, \cdots, 0\right)
$$


where the first $N_{0}$ entries are nonzero. This gives a color-flavor locking. Because of the quark mass matrix (2.4), the flavor symmetry of the original theory $S U\left(N_{f}\right) \times$ $U(1)^{\prime}$ is explicitly broken down to $O\left(N_{0}\right) \times U\left(N_{f}-N_{0}\right)$. Therefore the present vacuum manifold is quite different from the meta-stable vacuum manifold of [8, 9]. Our vacuum manifold is just a point ${ }^{\dagger}$ (times the space spanned by $\Phi_{0}$ ), and the symmetry of the vacuum is $S O\left(N_{0}\right)_{\mathrm{C}+\mathrm{F}} \times G$, where the first $S O\left(N_{0}\right)_{\mathrm{C}+\mathrm{F}}$ is the colorflavor locking symmetry, and $G \in U\left(N_{f}-N_{0}\right)$ is the symmetry preserved by $\Phi_{0}$ : for example if $\Phi_{0}=0, G=U\left(N_{f}-N_{0}\right)$. Accordingly, our situation is different from [13] where a "Seiberg-like" dual of semilocal vortex moduli space was studied. The vacuum has a modulus $\Phi_{0}$, which survives even in the limit of large $\mu$ to the confining phase.

In the case of $N=2$ in which the magnetic theory has $S O(2) \sim U(1)$ gauge group and so is in Abelian Coulomb phase, the superpotential (2.1) is a little modified [3] as

$$
W=h\left(a(t) \sum_{i, j=1}^{N_{f}} \Phi_{i j} q_{i}^{+} q_{j}^{-}-\mu^{2} \sum_{i=1}^{N_{0}} \Phi_{i i}\right) .
$$

Here $q_{i}^{ \pm}$are "monopoles" which possess electric $U(1)$ charges in the dual $S O(2) \sim$ $U(1)$ theory. In the superpotential, $t \equiv(\operatorname{det} \Phi) / \Lambda^{2\left(N_{c}-2\right)}$ and $a(0)=1$. The mass for the quarks in the electric theory was already chosen as (2.4) so that the vacuum is supersymmetric; so we have two choices, $N_{0}=1$ or $N_{0}=2$. The superpotential (2.7) looks different from (2.1), but in fact they are very similar to each other. If we redefine the matter chiral superfields as

$$
q_{i}^{+}=\varphi_{i}^{1}+i \varphi_{i}^{2}, \quad q_{i}^{-}=\varphi_{i}^{1}-i \varphi_{i}^{2}
$$

where the upper indices are for the $S O(2)$ vector representation, then (2.7) reduces to (2.1) except for the difference of the factor $a(t)$. For deriving the vacuum for $N=2$ with the choice of the quark mass matrix (2.4) with $N_{0}=N$, in fact this factor $a(t)$ is irrelevant, so the supersymmetric vacuum configuration is again (2.5). The dual quarks (which are the "monopoles") condense and the theory is in the Higgs phase with massive photons.

\section{Vortex string solution}

What we have shown in our previous paper [9] was that even in this vacua (2.5) with supersymmetries unbroken, there exists a non-BPS vortex string solution, for the case of $U\left(N_{c}\right)$ gauge groups. Here we explicitly generalize the study given there

\footnotetext{
${ }^{\dagger}$ Precisely speaking, the vacua consist of two points, $Z_{2}=O\left(N_{0}\right) / S O\left(N_{0}\right)$.
} 


\begin{tabular}{c|ccccccccc} 
NS & 1 & 2 & 3 & - & - & - & - & 8 & 9 \\
NS' & 1 & 2 & 3 & 4 & 5 & - & - & - & - \\
D6 & 1 & 2 & 3 & - & - & - & 7 & 8 & 9 \\
D4 & 1 & 2 & 3 & - & - & 6 & - & - & - \\
O4 & 1 & 2 & 3 & - & - & 6 & - & - & - \\
\hline D2 & - & - & 3 & 4 & - & - & - & - & -
\end{tabular}

Table 1: Brane configuration for the magnetic theory with $S O(N)$ gauge group. We add a D2-brane (the lowest row) to represent the vortex string.

to the theory with $S O\left(N_{c}\right)$ gauge groups, to relate the classical vortex strings with the QCD strings in confining gauge theories.

The existence of the non-BPS vortex string in the case of $S O(N)$ magnetic theory can be seen in its brane configuration. The $U(N)$ case was studied in our previous paper, and its generalization to the $S O(N)$ case is straightforward. The brane configuration representing the vacuum of the $S O(N)$ magnetic theory, derived by using the brane realization [14] of the Seiberg-duality in the Hanany-Witten configurations [15], was given in [16] and shown in the table 1. ${ }^{\ddagger}$ (Note that [16] studied the supersymmetry-breaking meta-stable vacua while we are interested in the quark mass alignment (2.4), so all the D4-branes are parallel to each other in our case, as studied in our previous paper [9] for the $U(N)$ case.) As in [9], we can add a D2-brane suspended between the D4-branes and the NS5-brane. This D2-brane is oriented along $x^{3}$ and $x^{4}$ directions. This is the vortex string we are interested in. The orientifold requires that a mirror D2-brane should be added properly. This brane realization of vortices is along the original idea of [18, 19].

So, string theory predicts the existence of a non-BPS vortex string solution in this magnetic $S O(N)$ theory. Being helped by this prediction, we are able to find an explicit solution of the vortex string. For the case of $S O(N)$ with $N>2$, the vortex string solution can be constructed by an embedding of the ANO string into an $S O(2)$ sub-sector in the $S O(N)$. For the case of $S O(2)$, we will find that the solution is in fact just a multiple-copied ANO solution.

For our purpose to show the dual counterpart of the QCD string, it is enough to consider one choice of $N$, so let us study the $S O(2)$ case which is the simplest. Furthermore we consider $N_{0}=N$ for the quark mass (2.4). Later we study the case of general $N$. The potential derived from the superpotential (2.7) and the D-term

\footnotetext{
${ }^{\ddagger}$ For $S U\left(N_{c}\right)$ case and its M-theory lift, see [17].
} 
potential are

$$
\begin{aligned}
V= & V_{F}+V_{D} \\
= & h^{2}\left|a(t) q_{1}^{+} q_{1}^{-}-\mu^{2}\right|^{2}+h^{2}\left|a(t) q_{2}^{+} q_{2}^{-}-\mu^{2}\right|^{2}+h^{2} \sum_{i=3}^{N_{f}}\left|a(t) q_{i}^{+} q_{i}^{-}\right|^{2} \\
& +\frac{1}{2} h^{2} a(t)^{2} \sum_{i \neq j}^{N_{f}}\left|q_{i}^{+} q_{j}^{-}+q_{j}^{+} q_{i}^{-}\right|^{2}+\frac{g^{2}}{2} \sum_{i=1}^{N_{f}}\left(\left|q_{i}^{+}\right|^{2}-\left|q_{i}^{-}\right|^{2}\right)^{2}+\mathcal{O}\left(\Phi^{2}\right) .
\end{aligned}
$$

Here we have omitted writing higher order terms in $\Phi$ because this field is kept being the vacuum (2.5) for obtaining the vortex string solution. So we can take $t=0$ and therefore $a(t)=1$.

We work in the convention with the monopoles $q_{i}^{ \pm}$rather than $\varphi_{i}$, because the former has direct relevance to the ANO solution, as we will find below. In terms of these monopole fields, the vacuum (2.5) is

$$
q_{1}^{+}=q_{1}^{-}=-i q_{2}^{+}=i q_{2}^{-}=\mu, \quad q_{i}^{ \pm}=0(i \geq 3)
$$

up to the $Z_{2} \in O\left(N_{F}\right)$. It is very natural that the ANO vortex string solution is embedded in the following manner:

$$
\begin{aligned}
& q_{1}^{+}=\left(q_{1}^{-}\right)^{*}=-i q_{2}^{+}=i\left(q_{2}^{-}\right)^{*}=f(r) e^{i n \theta}, \quad q_{i}^{ \pm}=0(i \geq 3), \\
& A_{\theta}=\frac{-n \alpha(r)}{g}, \quad A_{0}=A_{3}=0 .
\end{aligned}
$$

Here $r \equiv \sqrt{\left(x^{1}\right)^{2}+\left(x^{2}\right)^{2}}$ and $\theta \equiv \arctan \left(x^{2} / x^{1}\right)$ span the cylindrical coordinates with $x^{3}$. One can check that this is in fact a solution of the full system. The equations for the functions $f(r)$ and $\alpha(r)$ are

$$
\begin{aligned}
& \frac{d^{2}}{d r^{2}} f+\frac{1}{r} \frac{d}{d r} f-\frac{n^{2}}{r^{2}}(\alpha-1)^{2} f-h^{2}\left(f^{2}-\mu^{2}\right) f=0, \\
& \frac{d^{2}}{d r^{2}} \alpha-\frac{1}{r} \frac{d}{d r} \alpha-8 g^{2}(\alpha-1) f^{2}=0 .
\end{aligned}
$$

To derive this, we used the Kähler potential for the monopole fields as $K=\left(\left|q^{+}\right|^{2}+\right.$ $\left.\left|q^{-}\right|^{2}\right)$. The functions $f(r)$ and $\alpha(r)$ interpolate $f(0)=\alpha(0)=0$ and the vacuum with the winding, $f(\infty) / \mu=\alpha(\infty)=1$. This is the same as the famous ANO solution [1] (20]). See Figure 1]. The vortex string carries $n$ unit of the magnetic flux. The width of the vortex measured by the monopole scalar fields is $\sim h \mu$, while the width of the concentration of the magnetic flux is $\sim g \mu$. When $h=2 g$ (which is the BPS limit), the tension of the vortex string is given by $2 \pi \mu^{2}$. When $h$ differs from the BPS value $2 g$, the tension is roughly estimated as $\mathcal{O}\left(\mu^{2}\right)$.

The embedding ansatz is chosen so that it does not violate the vanishing of the crossing terms (the fourth and the fifth terms) in the potential (3.1). The winding 

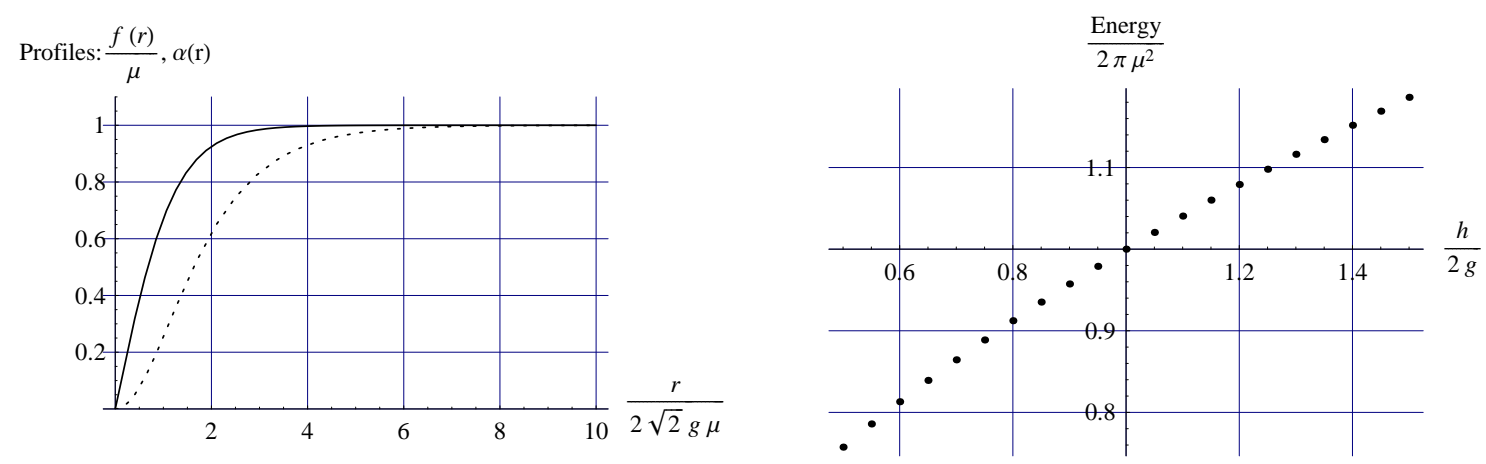

Figure 1: Left: The functions $f(r)$ (solid line) and $\alpha(r)$ (dashed line) versus $r$. Right: Coupling dependence of the vortex tension.

number is given by $n$, which is the magnetic flux of the vortex string. This is the dual of the QCD string, for the case of $S O\left(N_{c}\right)$ supersymmetric QCD with the $N_{f}=N_{c}-4$ quarks in the vector representation of $S O\left(N_{c}\right)$.

In the analysis above, we have chosen $N_{0}=2$. However, even with $N_{0}=1$, the electric theory is confined after the decoupling of this single massive quark [3], since $N_{f}=N_{c}-3$. Therefore we expect that a similar vortex string solution exists also for this $N_{0}=1$ and $N=2$. (This case of $N_{0}=1$ is what M. Strassler studied in his original discussion [10].) Let us present the solution. The potential is

$$
\begin{aligned}
V= & h^{2}\left|a(t) q_{1}^{+} q_{1}^{-}-\mu^{2}\right|^{2}+h^{2} \sum_{i=2}^{N_{f}}\left|a(t) q_{i}^{+} q_{i}^{-}\right|^{2} \\
& +\frac{1}{2} h^{2} a(t)^{2} \sum_{i \neq j}^{N_{f}}\left|q_{i}^{+} q_{j}^{-}+q_{j}^{+} q_{i}^{-}\right|^{2}+\frac{g^{2}}{2} \sum_{i=1}^{N_{f}}\left(\left|q_{i}^{+}\right|^{2}-\left|q_{i}^{-}\right|^{2}\right)^{2}+\mathcal{O}\left(\Phi^{2}\right) .
\end{aligned}
$$

The vacuum is unique: $q_{1}^{+}=q_{1}^{-}=\mu$ and the other fields are zero. The vortex string solution is

$$
q_{1}^{+}=\left(q_{1}^{-}\right)^{*}=f(r) e^{i n \theta}, \quad A_{\theta}=\frac{-n \alpha(r)}{g}, \quad A_{0}=A_{3}=0, \quad q_{i>1}^{ \pm}=0 .
$$

This is the dual of the QCD string, for the case of $N_{f}=N_{c}-3$. A similar solution can be easily constructed around the massless dyon point [3] in the moduli space. But this point flows to a runaway vacuum at low energy, and so the vortex string is irrelevant to the QCD string.

Next, we study the generic case of $S O(N)$. The vacuum (2.5) breaks the $S O(N)$ gauge group completely and we expect that there are non-Abelian strings with the $Z_{2}\left(=\pi_{1}(S O(N))\right)$ charge. We choose $N_{0}=N$ to make sure the confining phase at low energy of the electric theory. (One can choose $N_{0}=N-1$ alternatively, as in the case of $N=2$ above, but the solution is the same as the one presented below.) 
Without losing generality, we can choose the embedding of the $S O(2)$ as just the first two raws and columns of the $S O(N) . \S$ Then, relevant fields have the potential terms

$$
\begin{aligned}
V_{F}+V_{D}= & h^{2}\left|\left(\varphi_{1}^{a}\right)^{2}-\mu^{2}\right|^{2}+h^{2}\left|\left(\varphi_{2}^{a}\right)^{2}-\mu^{2}\right|^{2}+4 h^{2}\left|\varphi_{1}^{a} \varphi_{2}^{a}\right|^{2} \\
& +\frac{g^{2}}{8}\left(\left(\varphi_{1}^{a}\right)^{*} \epsilon_{a b} \varphi_{1}^{b}+\left(\varphi_{2}^{a}\right)^{*} \epsilon_{a b} \varphi_{2}^{b}\right)^{2} .
\end{aligned}
$$

The terms involving the fields $\varphi_{i}$ with $i \geq 3$ and $\Phi$ are omitted since they are irrelevant. For the D-term to be trivially satisfied, we turn on only the real part of the fields. Then we combine the nontrivial real part of the fields as

$$
\widetilde{\varphi}_{1} \equiv \operatorname{Re} \varphi_{1}^{1}+i \operatorname{Re} \varphi_{1}^{2}, \quad \widetilde{\varphi}_{2} \equiv \operatorname{Re} \varphi_{2}^{1}+i \operatorname{Re} \varphi_{2}^{2}
$$

The $S O(2)$ acts as a $U(1)$ phase gauge rotation on these complex scalar fields, and therefore the following embedding of the ANO solution works,

$$
\widetilde{\varphi}_{1}=f(r) e^{i n \theta}, \quad \widetilde{\varphi}_{2}=i f(r) e^{i n \theta} .
$$

The relative phase $i$ in the above embedding is chosen so that the cross-term $\left|\varphi_{1}^{a} \varphi_{2}^{a}\right|^{2}$ of the F-term potential in (3.8) vanishes. It can be shown straightforwardly that this embedding is a solution of the whole system, when the other components of the fields are chosen to be those of the vacuum. Note that the solution is the same as the solution (3.3) for the $N=2$ case though in different notations and we can write (3.10) as

$$
\left(\begin{array}{ll}
\varphi_{1}^{1} & \varphi_{1}^{2} \\
\varphi_{2}^{1} & \varphi_{2}^{2}
\end{array}\right)=f(r)\left(\begin{array}{cc}
\cos (n \theta) & \sin (n \theta) \\
-\sin (n \theta) & \cos (n \theta)
\end{array}\right) .
$$

We have a choice of how to embed the $S O(2)$ in the whole $S O(N)$. This should provide an orientational moduli of the vortex string, as in the famous examples of the $U(N)$ non-Abelian vortex strings [18, 5]. The freedom of this choice can be seen in the brane configuration: the D2-brane can choose one D4-branes among $N$ of them, to end. ${ }^{\text {In }}$ the large $\mu$ limit, this moduli space is expected to shrink and reduce to a point, because the confining theory in the electric side doesn't know which $N$ one has started with before taking the limit.

\section{Stability of the vortex string solution}

The vortex string solution obtained should be stable, because at the low energy the electric theory is in the confining phase and so the flux tube does not decay by broadening itself. However, the classical system of the dual theory which admits the vortex

\footnotetext{
${ }^{\S}$ Similar kinds of vortex solutions in $S O(N)$ gauge theories have been constructed and studied in [21, 田.

The actual orientational moduli is continuous while the choice of a D4-brane is discrete: the brane configuration would show only the information of the Cartan sub-algebra.
} 
string solutions as above looks similar to the one which admits so-called semilocal strings [22], since our dual theory has $N_{f}>N$. It is known that the semilocal strings are unstable and develop tachyonic instability for a particular parameter region of the theory. Actually, the vortex strings in the meta-stable vacua studied in [9] have such instability. Here we show that, on the contrary to the expectation from this similarity, our vortex string solution is stable classically. Our vortex string is not semilocal" , and in particular for $N=2$ it has no moduli space (except for the $\Phi_{0}$ degree of freedom).

Let us concentrate on the example of $S O(2)$ with $N_{0}=2$, in which the solution is given by (3.3). (The system is in fact very similar to the one derived from $\mathcal{N}=2$ theory.) Fluctuation analysis is easier with the following variables

$$
\phi_{1} \equiv \frac{1}{\sqrt{2}}\left(q_{1}^{+}+\left(q_{1}^{-}\right)^{\dagger}\right), \quad \widetilde{\phi}_{1} \equiv \frac{1}{\sqrt{2}}\left(q_{1}^{+}-\left(q_{1}^{-}\right)^{\dagger}\right)
$$

and similar definition for $\phi_{2}$ and $\widetilde{\phi}_{2}$ from $q_{2}^{ \pm}$. The solution lives in the $\phi_{1}, \phi_{2}$ sector since (3.3) gives $\widetilde{\phi}_{1}=\widetilde{\phi}_{2}=0$. The solution is stable against fluctuations of $\phi_{i}$ since the analysis is just the same as the Abelian-Higgs model. So, let us turn on the fluctuation $\widetilde{\phi}_{i}$. The potential can be expanded to the second order in $\widetilde{\phi}_{1}$ as

$\frac{h^{2}}{4}\left(\left|\phi_{1}\right|^{2}-2 \mu^{2}\right)^{2}+\frac{h^{2}}{4}\left|\phi_{1} \widetilde{\phi}_{1}^{\dagger}-\phi_{1}^{\dagger} \widetilde{\phi}_{1}\right|^{2}+\frac{h^{2}}{2}\left(2 \mu^{2}-\left|\phi_{1}\right|^{2}\right)\left|\widetilde{\phi}_{1}\right|^{2}+\frac{g^{2}}{2}\left(\phi_{1} \widetilde{\phi}_{1}^{\dagger}+\widetilde{\phi}_{1} \phi_{1}^{\dagger}\right)^{2}$.

Because $f(r)<\mu$ for $r<\infty$, this is positive semi-definite, and so is the potential for fluctuation of $\widetilde{\phi}_{2}$. The remaining terms relevant in the potential (3.1) are the third and the fourth terms in (3.1), but it is obvious that they are already of the second order in fluctuations $\widetilde{\phi}_{i}$ and $q_{i>2}^{ \pm}$, so they are positive semi-definite. We conclude that our vortex string solution (3.3) is stable and has no moduli space except for massless modes associated with $\Phi_{0}$. The stability of the solution (3.7) can be shown in the same manner.

In our topological argument in [9], there are only $Z_{2}$ strings for the case of $N>2$, in contrast to the case of $S O(2)$ where the winding number $n \in Z$ is the topological charge. We expect that the $S O(N)$ vortex solutions with higher winding numbers we constructed are meta-stable in this sense. ${ }^{* *}$ There may be no topological obstacle to deform the vortex configuration with a higher winding number to that with a lower one by the $Z_{2}$ grading, but there may be a potential barrier. The solutions in the case of $S O(2)$ and the solutions in $S O(N)$ should be somehow related by a mass deformation of the quarks in the original electric theory. One can change $N$ by changing $N_{f}$ while $N_{c}$ being fixed. In this sense, the "meta-stable" solutions (3.10) with higher winding numbers look rather natural.

\footnotetext{
"When $N_{0}>N$, the perturbative vacuum is supersymmetry-breaking and meta-stable, and the vacuum moduli space is non-trivial. The vortex strings living there are semilocal, as shown in [9]. The semilocality and its relevance to the confinement was discussed in [23, 7].

${ }^{* *}$ Similar discussions for $S U\left(N_{c}\right)$ QCD and its $Z_{N_{c}}$ strings can be found in [11, 24].
} 
Note added: While we were writing this paper, we became aware of the paper 25] which discusses relevance of our vortex string found in [9] to a QCD string.

\section{Acknowledgments}

M.E. would like to thank M. Nitta and W. Vinci for discussions. K.H. is grateful to N. Yokoi for helpful discussions, and would like to thank Y. Kikukawa and T. Yoneya for useful comments. The work of M.E. is supported by Japan Society for the Promotion of Science under the Post-doctoral Research Program Abroad. K.H. and S.T. are partly supported by the Japan Ministry of Education, Culture, Sports, Science and Technology.

\section{References}

[1] A. A. Abrikosov, "On the Magnetic properties of superconductors of the second group," Sov. Phys. JETP 5, 1174 (1957) [Zh. Eksp. Teor. Fiz. 32, 1442 (1957)];

H. B. Nielsen and P. Olesen, "Vortex-Line Models for Dual Strings," Nucl. Phys. B 61, 45 (1973).

[2] N. Seiberg, "Electric - magnetic duality in supersymmetric nonAbelian gauge theories," Nucl. Phys. B 435 (1995) 129 [hep-th/9411149].

[3] K. A. Intriligator and N. Seiberg, "Duality, monopoles, dyons, confinement and oblique confinement in supersymmetric $S O(N(c))$ gauge theories," Nucl. Phys. B 444, 125 (1995) [arXiv:hep-th/9503179].

[4] K. Konishi and L. Spanu, "Non-Abelian vortex and confinement," Int. J. Mod. Phys. A 18, 249 (2003) [arXiv:hep-th/0106175].

[5] R. Auzzi, S. Bolognesi, J. Evslin, K. Konishi and A. Yung, "Nonabelian superconductors: Vortices and confinement in $N=2 S Q C D$," Nucl. Phys. B 673 (2003) 187 [hep-th/0307287].

[6] R. Auzzi, S. Bolognesi, J. Evslin and K. Konishi, "Nonabelian monopoles and the vortices that confine them," Nucl. Phys. B 686, 119 (2004) [arXiv:hep-th/0312233];

A. Gorsky, M. Shifman and A. Yung, "Non-Abelian Meissner effect in Yang-Mills theories at weak coupling," Phys. Rev. D 71, 045010 (2005) [arXiv:hep-th/0412082];

M. Eto, L. Ferretti, K. Konishi, G. Marmorini, M. Nitta, K. Ohashi, W. Vinci, N. Yokoi, "Non-Abelian duality from vortex moduli: a dual model of color-confinement," arXiv:hep-th/0611313;

M. R. Douglas and S. H. Shenker, Nucl. Phys. B 447 (1995) 271 [arXiv:hep-th/9503163]; 
[7] For a recent review, M. Shifman and A. Yung, "Supersymmetric Solitons and How They Help Us Understand Non-Abelian Gauge Theories," arXiv:hep-th/0703267.

[8] K. Intriligator, N. Seiberg and D. Shih, "Dynamical SUSY breaking in meta-stable vacua," JHEP 0604 (2006) 021 [hep-th/0602239].

[9] M. Eto, K. Hashimoto and S. Terashima, "Solitons in supersymmety breaking meta-stable vacua," JHEP 0703, 061 (2007) [arXiv:hep-th/0610042].

[10] M. J. Strassler, "Duality, phases, spinors and monopoles in $S O(N)$ and $\operatorname{spin}(N)$ gauge theories," JHEP 9809, 017 (1998) [arXiv:hep-th/9709081].

[11] M. J. Strassler, "Messages for QCD from the superworld," Prog. Theor. Phys. Suppl. 131, 439 (1998) [arXiv:hep-lat/9803009].

[12] M. J. Strassler, "On phases of gauge theories and the role of non-BPS solitons in field theory," arXiv:hep-th/9808073.

[13] M. Eto, J. Evslin, K. Konishi, G. Marmorini, M. Nitta, K. Ohashi, W. Vinci and N. Yokoi, "On the moduli space of semilocal strings and lumps," arXiv:0704.2218 [hep-th].

[14] K. Landsteiner, E. Lopez and D. A. Lowe, Nucl. Phys. B 507 (1997) 197 [arXiv:hep-th/9705199];

S. Elitzur, A. Giveon, D. Kutasov, E. Rabinovici and A. Schwimmer, Nucl. Phys. B 505 (1997) 202 [arXiv:hep-th/9704104];

H. Ooguri and C. Vafa, Nucl. Phys. B 500 (1997) 62 [arXiv:hep-th/9702180].

[15] A. Hanany and E. Witten, "Type IIB superstrings, BPS monopoles, and three-dimensional gauge dynamics,” Nucl. Phys. B 492 (1997) 152 [hep-th/9611230];

S. Elitzur, A. Giveon and D. Kutasov, "Branes and $N=1$ duality in string theory," Phys. Lett. B 400 (1997) 269 [hep-th/9702014];

For a review, A. Giveon and D. Kutasov, "Brane dynamics and gauge theory," Rev. Mod. Phys. 71 (1999) 983 [hep-th/9802067].

[16] S. Franco, I. Garcia-Etxebarria and A. M. Uranga, "Non-supersymmetric meta-stable vacua from brane configurations," JHEP 0701, 085 (2007) [arXiv:hep-th/0607218];

C. Ahn, "Meta-Stable Brane Configurations by Adding an Orientifold-Plane to Giveon-Kutasov," arXiv:0706.0042 [hep-th].

[17] H. Ooguri and Y. Ookouchi, "Meta-stable supersymmetry breaking vacua on intersecting branes," Phys. Lett. B 641, 323 (2006) [arXiv:hep-th/0607183]; 
I. Bena, E. Gorbatov, S. Hellerman, N. Seiberg and D. Shih, "A note on (meta)stable brane configurations in MQCD," JHEP 0611, 088 (2006) [arXiv:hep-th/0608157].

[18] A. Hanany and D. Tong, "Vortex strings and four-dimensional gauge dynamics," JHEP 0404 (2004) 066 [hep-th/0403158]; "Vortices, instantons and branes," JHEP 0307 (2003) 037, [hep-th/0306150].

[19] B. H. Lee, H. j. Lee, N. Ohta and H. S. Yang, "Maxwell Chern-Simons solitons from type IIB string theory," Phys. Rev. D 60, 106003 (1999) [arXiv:hep-th/9904181].

[20] L. Jacobs and C. Rebbi, "Interaction Energy Of Superconducting Vortices," Phys. Rev. B 19, 4486 (1979).

[21] H. J. de Vega, "Fermions And Vortex Solutions In Abelian And Nonabelian Gauge Theories," Phys. Rev. D 18, 2932 (1978);

H. J. de Vega and F. A. Schaposnik, "Electrically Charged Vortices In Nonabelian Gauge Theories With Chern-Simons Term," Phys. Rev. Lett. 56, 2564 (1986); "Vortices and electrically charged vortices in nonAbelian gauge theories," Phys. Rev. D 34, 3206 (1986);

J. Heo and T. Vachaspati, "Z(3) strings and their interactions," Phys. Rev. D 58, 065011 (1998) [arXiv:hep-ph/9801455];

F. A. Schaposnik and P. Suranyi, "New vortex solution in SU(3) gauge-Higgs theory," Phys. Rev. D 62, 125002 (2000) [arXiv:hep-th/0005109];

V. Markov, A. Marshakov and A. Yung, "Non-Abelian vortices in $N=1^{*}$ gauge theory," Nucl. Phys. B 709, 267 (2005) [arXiv:hep-th/0408235];

L. Ferretti and K. Konishi, "Duality and confinement in $S O(N)$ gauge theories," arXiv:hep-th/0602252.

[22] T. Vachaspati and A. Achucarro, "Semilocal cosmic strings," Phys. Rev. D 44 (1991) 3067;

M. Hindmarsh, "Existence and stability of semilocal strings," Phys. Rev. Lett. 68 (1992) 1263;

A. Achucarro, K. Kuijken, L. Perivolaropoulos and T. Vachaspati, "Dynamical simulations of semilocal strings," Nucl. Phys. B 388 (1992) 435;

G. W. Gibbons, M. E. Ortiz, F. Ruiz Ruiz and T. M. Samols, "Semilocal strings and monopoles," Nucl. Phys. B 385 (1992) 127 [hep-th/9203023];

R. A. Leese and T. M. Samols, "Interaction of semilocal vortices," Nucl. Phys. B 396 (1993) 639.

[23] M. Shifman and A. Yung, "Non-Abelian semilocal strings in $N=2$ supersymmetric QCD," Phys. Rev. D 73, 125012 (2006) [arXiv:hep-th/0603134]. 
[24] M. Shifman and A. Yung, "Metastable strings in Abelian Higgs models embedded in non-Abelian theories: Calculating the decay rate," Phys. Rev. D 66, 045012 (2002) [arXiv:hep-th/0205025];

A. Armoni and M. Shifman, "Remarks on stable and quasi-stable k-strings at large N,” Nucl. Phys. B 671, 67 (2003) [arXiv:hep-th/0307020];

K. Konishi, G. Marmorini, W. Vinci and N. Yokoi, in preparation.

[25] M. Shifman and A. Yung, "Confinement in N=1 SQCD: One Step Beyond Seiberg's Duality," arXiv:0705.3811 [hep-th]. 